OPEN ACCESS

Edited by: Jun Zhao,

Fudan University, China

Reviewed by:

Hechang Lei,

Renmin University of China, China

Masahiro Ishigami,

University of Central Florida,

United States

${ }^{*}$ Correspondence:

Xiaoli Dong

dong@iphy.ac.cn

Specialty section:

This article was submitted to Condensed Matter Physics,

a section of the journal

Frontiers in Physics

Received: 22 July 2020 Accepted: 16 September 2020 Published: 11 November 2020

Citation:

Dong X, Zhou F and Zhao Z (2020) Electronic and Superconducting Properties of Some FeSe-Based

Single Crystals and Films

Grown Hydrothermally.

Front. Phys. 8:586182.

doi: 10.3389/fphy.2020.586182

\section{Electronic and Superconducting Properties of Some FeSe-Based Single Crystals and Films Grown Hydrothermally}

\author{
Xiaoli Dong ${ }^{1,2,3 *}$, Fang Zhou ${ }^{1,2,3}$ and Zhongxian Zhao ${ }^{1,2,3}$ \\ ${ }^{1}$ Beijing National Laboratory for Condensed Matter Physics and Institute of Physics, Chinese Academy of Sciences, Beijing, \\ China, ${ }^{2}$ University of Chinese Academy of Sciences, Beijing, China, ${ }^{3}$ Songshan Lake Materials Laboratory, Dongguan, China
}

Our recent year's studies of the prototypal FeSe and molecule-intercalated (Li,Fe)OHFeSe superconductor systems are briefly reviewed here, with emphasis on the link between the superconducting and normal-state properties observed in the single crystals and films. These samples were successfully synthesized by our recently developed soft-chemical hydrothermal methods, which are also briefly described. Particularly in the Mn-doped high$T_{\mathrm{c}}$ (Li,Fe)OHFeSe film, a strong enhancement of the superconducting critical current density was achieved, which is promising for practical application of the superconductivity.

Keywords: superconductivity of iron selenides, normal state properties, electronic phase separation, spin nematicity, high critical current density, hydrothermal growth

\section{INTRODUCTION}

Iron-based superconductors [1] have received extensive attention because of their rich physics, including magnetic and nematic instabilities, electronic correlations, and quantum phenomena [2-9]. As the second class of high- $T_{c}$ materials after the discovery of cuprate superconductors, the iron-based superconductors are also promising for practical application owing to their large critical current density, high upper critical field, and small anisotropy [10-17]. The recent observation of Majorana zero modes in iron-based superconductors implies a potentiality for future application in topological quantum calculating [18-21]. Unlike an electronic configuration of $\mathrm{Cu}-3 \mathrm{~d}^{9}$ in the cuprates, the iron-based compounds have an electronic configuration of $\mathrm{Fe}-3 \mathrm{~d}^{6}$ and a small crystal-field splitting [2, 7, 22-24]. An immediate consequence of this is that all the five Fe-3d orbitals could be involved in the low-energy interactions [25], giving rise to the multiband nature of the iron-based superconductivity, and the complexity and multiplicity of the normal-state properties. The iron-based family has two major subclasses, the iron chalcogenide and pnictide superconductors. Among them, the iron selenide superconductors have been shown to display a highly tunable superconducting critical $T_{\mathrm{c}}$ and unique electronic properties in the normal state, thus providing a superior platform to investigate the underlying physics for iron-based superconductivity.

Superconductivity of FeSe-based compounds emerges from the edge-sharing FeSe-tetrahedra blocks, each formed by one iron-plane sandwiched between two selenium-planes. An important feature is that the superconducting $T_{\mathrm{c}}$ can be tuned in a wide range. The simplest binary FeSe shows bulk superconductivity at a lower $T_{\mathrm{c}} \sim 9 \mathrm{~K}$ under ambient pressure [26]. It is notable that $T_{\mathrm{c}}$ can be boosted to tens of kelvin $(30-50 \mathrm{~K})$, by the applications of high pressure [27-33], charge-carrier injection [34], electrochemical etching [35], and chemical intercalation. The weak van der Waals 
bonding between the neighboring FeSe-blocks allows a variety of FeSe-based intercalates to be obtained, such as the atomintercalated $\mathrm{A}_{\mathrm{y}} \mathrm{Fe}_{2-\mathrm{x}} \mathrm{Se}_{2}$ ( $\mathrm{A}=$ alkali metal) [36-40], moleculeintercalated $\left(\mathrm{Li}_{0.8} \mathrm{Fe}_{0.2}\right) \mathrm{OHFeSe}$ [41], and atom/molecule-cointercalated $\mathrm{Li}_{\mathrm{x}}\left(\mathrm{C}_{5} \mathrm{H}_{5} \mathrm{~N}\right)_{\mathrm{y}} \mathrm{Fe}_{2-\mathrm{z}} \mathrm{Se}_{2}$ [42], $\mathrm{A}_{\mathrm{x}}\left(\mathrm{NH}_{2}\right)_{\mathrm{y}}\left(\mathrm{NH}_{3}\right)_{1-\mathrm{y}} \mathrm{Fe}_{2} \mathrm{Se}_{2}$ $[43,44], \mathrm{A}_{\mathrm{x}}\left(\mathrm{NH}_{3}\right)_{\mathrm{y}} \mathrm{Fe}_{2} \mathrm{Se}_{2}$ [45-47] and $\mathrm{A}_{\mathrm{x}}\left(\mathrm{C}_{2} \mathrm{H}_{8} \mathrm{~N}_{2}\right)_{\mathrm{y}} \mathrm{Fe}_{2} \mathrm{Se}_{2}$ [48]. Moreover, the highest superconducting gap opening temperature $(\sim 65 \mathrm{~K})$ among all the iron-based superconductors has been observed in a monolayer FeSe grown on a $\mathrm{SrTiO}_{3}$ substrate $[49,50]$. On the other hand, distinct from most iron-based superconductor systems, FeSe does not order magnetically at ambient pressure, whereas a unique electronic nematic ordering has been observed to develop with a rotational-symmetrybreaking transition from a tetragonal to an orthorhombic phase at $T_{s} \sim 90 \mathrm{~K}[51,52]$. The electronic nematicity is directly related to a degeneracy lifting of the bands with $\mathrm{Fe}$ $3 \mathrm{~d}_{\mathrm{xz}}$ and $3 \mathrm{~d}_{\mathrm{yz}}$ orbital characters [53-55]. Compared to the Fermi-surface topology of the prototypal $\mathrm{FeSe}$, in the molecule-intercalated $(\mathrm{Li}, \mathrm{Fe}) \mathrm{OHFeSe}$ single crystals, only the electron pockets near the Brillouin zone corners are observed, in absence of the hole pocket near the zone center [56,57]. This raises question about a proposed pairing scenario of the electronic scatterings between the hole-like and electron-like pockets. Study of the FeSe-based superconductors is essential for a better understanding of the unconventional superconductivity.

To investigate the link between the unconventional superconductivity and unusual normal-state electronic properties, and the potential for the superconductivity application, high-quality single crystal and film samples are highly demanded. Recent years, we have been exploring softchemical methods suitable for synthesizing the FeSe-based superconductor single crystals and single-crystalline films hard to obtain by conventional high-temperature growth. By developing hydrothermal ion-exchange [58-60] and iondeintercalation [61, 62] approaches, we have succeeded in synthesizing series of high-quality sizable single crystals of the intercalated $(\mathrm{Li}, \mathrm{Fe}) \mathrm{OHFeSe}$ and binary FeSe systems, respectively. Our further study [9] has shown a strong electronic twodimensionality and a nearly linear extracted magnetic susceptibility in the hydrothermal high- $T_{\mathrm{c}}(42 \mathrm{~K})(\mathrm{Li}, \mathrm{Fe})$ OHFeSe single crystal, suggesting the presence of twodimensional magnetic fluctuations in the normal state. In a series of the ( $\mathrm{Li}, \mathrm{Fe}) \mathrm{OHFeSe}$ single crystals, a coexistence of antiferromagnetism with superconductivity has been detected [60]. We explain such coexistence by electronic phase separation, similar to the previously observed in high- $T_{\mathrm{c}}$ cuprates and iron arsenides. An electronic phase diagram is
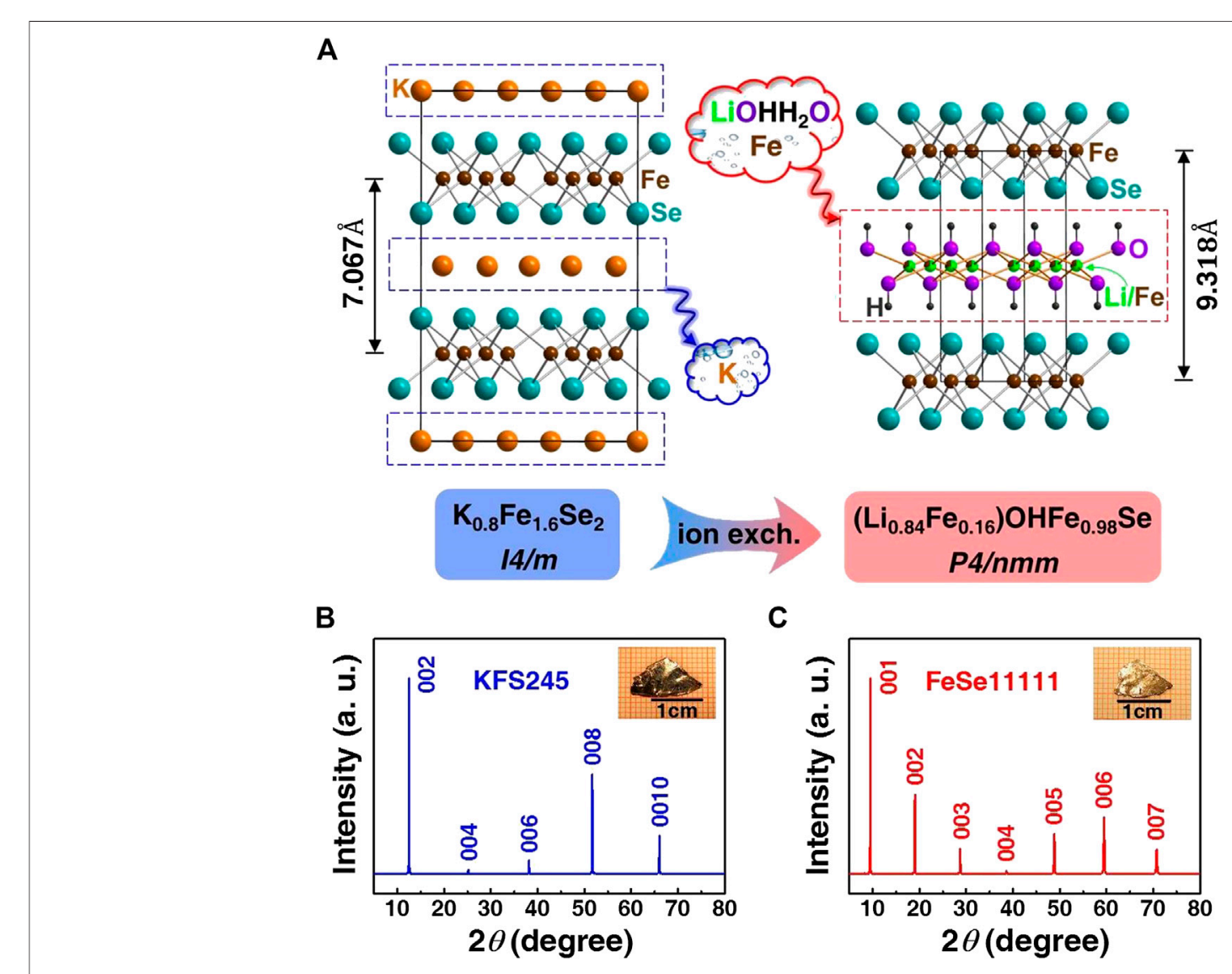

FIGURE 1 | Illustration of hydrothermal ion-exchange growth of (Li,Fe)OHFeSe crystals [58]. 
further established for ( $\mathrm{Li}, \mathrm{Fe}$ )OHFeSe system [60, 63]. In hydrothermal binary $\mathrm{Fe}_{1-x} \mathrm{Se}$ single crystals, we have observed a field-induced two-fold rotational symmetry emerging below $T_{\text {sn }}$ in angular-dependent magnetoresistance measurements, and a linear relationship between $T_{\mathrm{c}}$ and $T_{\mathrm{sn}}[61,64]$. Importantly, we find in our recent study [9] that the superconductivity of FeSe system emerges from the strongly correlated, hole-dominated $\mathrm{Fe}_{1-x} \mathrm{Se}$ as the non-stoichiometry is reduced to $x \sim 5.3 \%$. Interestingly, such an $x$ threshold for superconductivity of the prototypal FeSe is similar to that $\left(x \sim 5 \%\right.$ [65]) for high- $T_{\mathrm{c}}$ superconductivity of the intercalated ( $\mathrm{Li}, \mathrm{Fe}$ )OHFeSe sharing the common superconducting FeSe-blocks.

We have also successfully synthesized a series of high-quality single-crystalline films of ( $\mathrm{Li}, \mathrm{Fe}) \mathrm{OHFeSe}$ system, by inventing a hydrothermal epitaxial film technique $[16,17,66]$. We find that doping $\mathrm{Mn}$ into high- $T_{\mathrm{c}}(\mathrm{Li}, \mathrm{Fe}) \mathrm{OHFeSe}$ films can raise the superconducting critical current density $J_{c}$ by one order of magnitude to $0.32 \mathrm{MA} / \mathrm{cm}^{2}$ at a high field of $33 \mathrm{~T}$ [17]. Such a high $J_{c}$ value is the record so far among the iron-based superconductors, and is thus promising for high-field application of the superconductivity. Besides, our breakthrough in the crystal growth has greatly promoted other related studies and progresses have been made $[57,59,60,67-71]$, including the ARPES study of Fermi-surface topology [57] and the observation of pressure-induced second high- $T_{\mathrm{c}}(>50 \mathrm{~K})$ phase [70] in the (Li,Fe)OHFeSe system. Our developed growth method has also been adopted in the studies of other research groups [56, 72-83].

\section{SOFT-CHEMICAL HYDROTHERMAL GROWTH METHODS DEVELOPED FOR FESE-BASED SINGLE CRYSTALS AND FILMS}

The discovery of $\mathrm{Li}_{0.8} \mathrm{Fe}_{0.2} \mathrm{OHFeSe}$ (FeSe-11111) superconductor [41] brings new opportunity for the study of iron-based superconductivity. ( $\mathrm{Li}, \mathrm{Fe}) \mathrm{OHFeSe}$ is free from the complications of the structural transition, associated with the electronic nematicity, and the chemical phase separation, related to the intergrown insulating $\mathrm{K}_{0.8} \mathrm{Fe}_{1.6} \mathrm{Se}_{2}$ (KFS-245 phase) [63], as compared to the prototypal FeSe-11 and $\mathrm{K}_{1-\mathrm{y}} \mathrm{Fe}_{2-\mathrm{x}} \mathrm{Se}_{2}-122$ superconductors, respectively. Moreover, it shows an ambient-pressure high $T_{\mathrm{c}}=$ $42 \mathrm{~K}$ and a pressure-induced higher $T_{\mathrm{c}}>50 \mathrm{~K}$ under $12.5 \mathrm{GPa}$ [70]. Having a Fermi-surface topology $[56,57]$ similar to the high- $T_{\mathrm{c}}(>65 \mathrm{~K})$ FeSe monolayer, (Li, Fe)OHFeSe system turns out to be an ideal platform for studying the superconducting and normal-state properties of high- $T_{\mathrm{c}}$ iron-based superconductors. Initially, only the powder samples of ( $\mathrm{Li}, \mathrm{Fe}) \mathrm{OHFeSe}$ can be prepared hydrothermally $[41,63,65,84,85]$. For in-depth investigations on the intrinsic and anisotropic physical properties, the high-quality single crystal and film samples are indispensable.

The crystal structure of ( $\mathrm{Li}, \mathrm{Fe}) \mathrm{OHFeSe}$ consists of a stacking of one superconducting (SC) FeSe-block alternating with one insulating ( $\mathrm{Li}, \mathrm{Fe}) \mathrm{OH}$-block along the $c$-axis. The $(\mathrm{Li}, \mathrm{Fe}) \mathrm{OHFeSe}$ compound suffers an easy decomposition because of the inherent

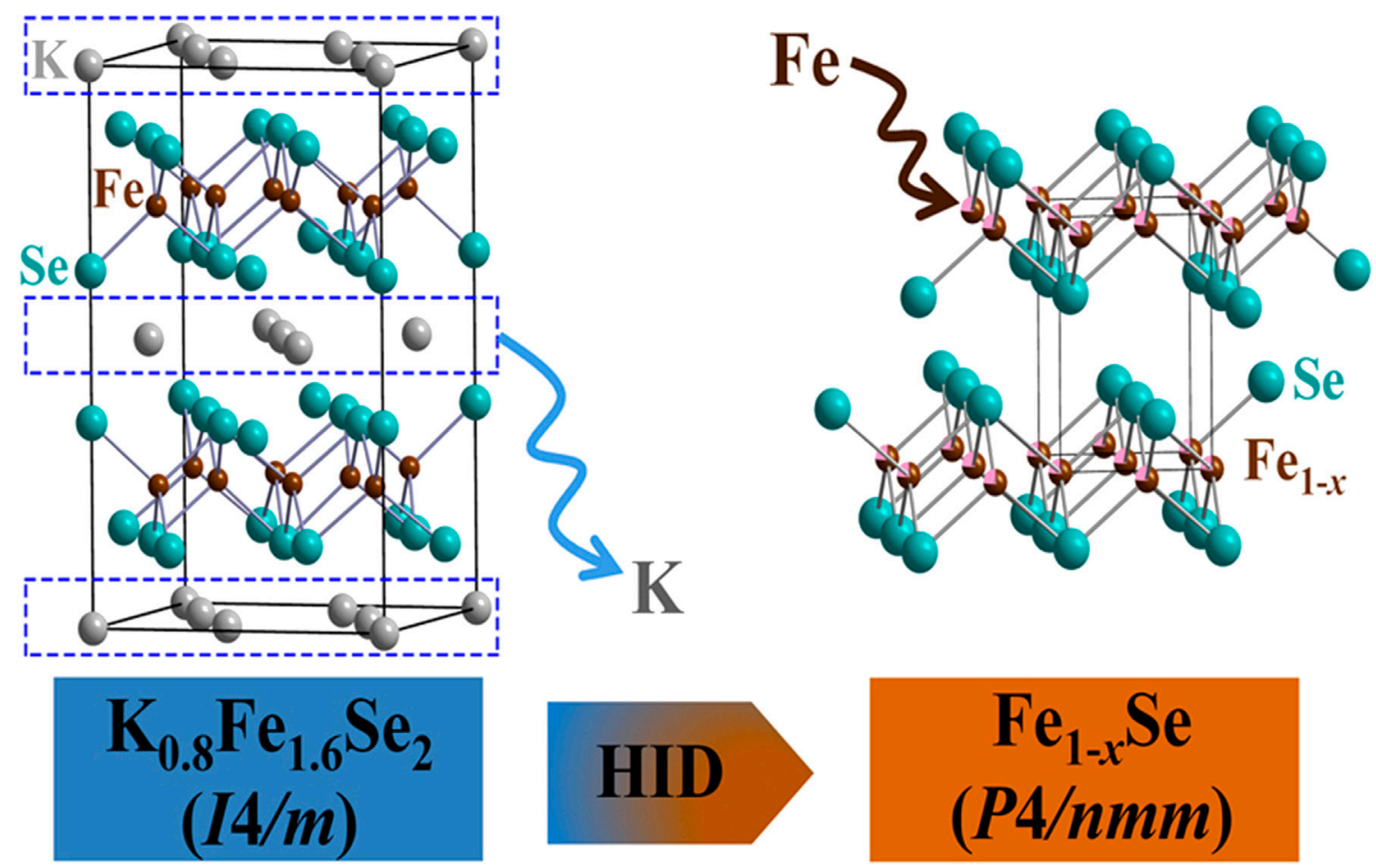

FIGURE 2 | Scematic llutration of the hydrothermal ion-deintercalation method. During the HID process, Fe $\mathrm{e}_{1-x} \mathrm{Se}$ single crystals are derived from the readily obtainable phase-pure matrix single crystals of $\mathrm{K}_{0.8} \mathrm{Fe}_{1.6} \mathrm{Se}_{2}$. The original interlayer $\mathrm{K}$ ions and $\mathrm{Fe}$ vacancies (20\% in amount) in $\mathrm{K}_{0.8} \mathrm{Fe}_{1.6} \mathrm{Se}_{2}$ were completely de-intercalated and substantially reduced, respectively, yielding the target single crystals of phase-pure $\mathrm{Fe}_{1-x} \mathrm{Se}^{2}$, 61, 62]. 


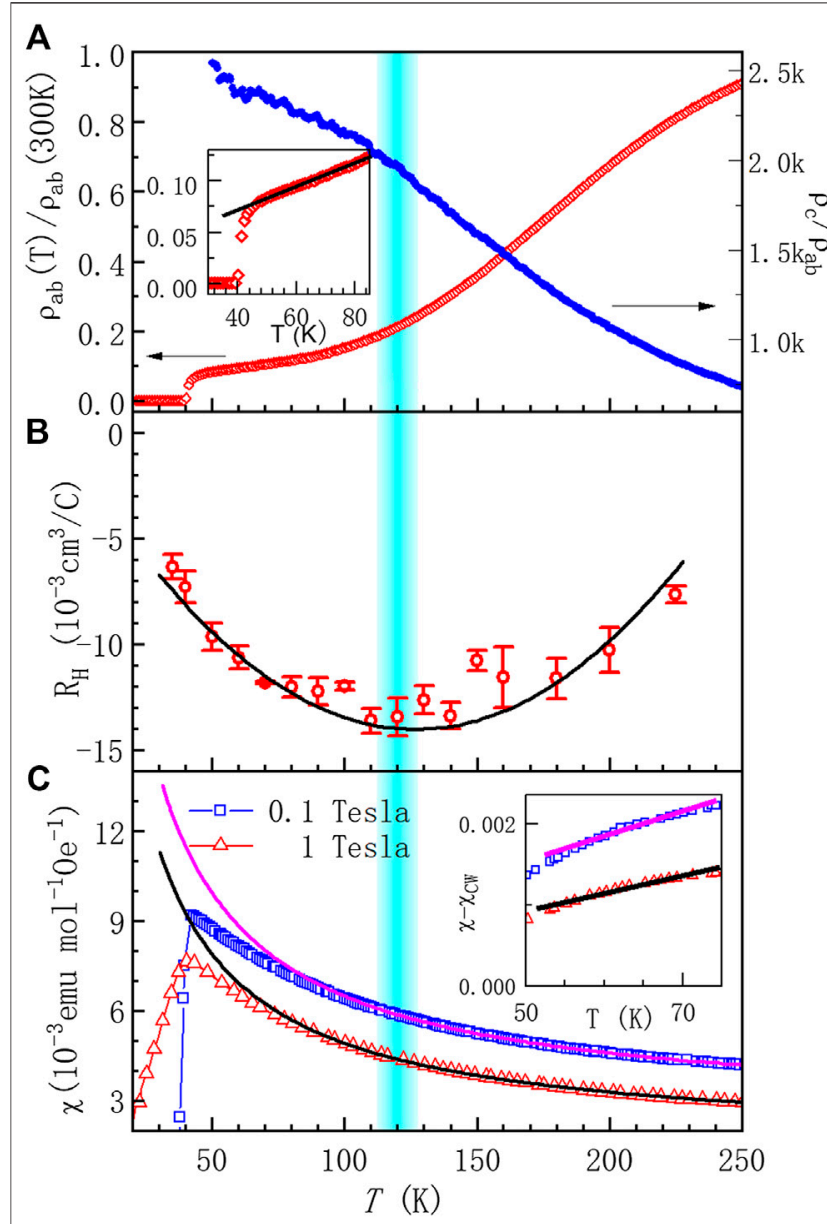

FIGURE 3 | The electrical transport and magnetic properties of $\left(\mathrm{Li}_{0.84} \mathrm{Fe}_{0.16}\right) \mathrm{OHFe}_{0.98} \mathrm{Se}$ single crystal [58]. (A) The in-plane electric resistivity and the ratio of out-of-plane to in-plane resistivity as functions of temperature. The inset shows the linear resistivity below the Hall-dip temperature $T^{*}$ down to $T_{\mathrm{c}}$. (B) The temperature dependence of in-plane Hall coefficient shows a dip-like feature around $T^{\star} \sim 120 \mathrm{~K}$. (C) The temperature dependencies of static magnetic susceptibility under magnetic fields along $c$-axis. A deviation from the Curie-Weiss law is clearly visible below the Hall-dip temperature $T^{\star}$. After subtracting the Curie-Weiss term (the solid fitted curves) from the $\left(\mathrm{Li}_{0.84} \mathrm{Fe}_{0.16}\right) \mathrm{OH}$-blocks, a nearly linear magnetic susceptibility from the FeSe-blocks is obvious (the inset).

weak hydrogen bonding. Therefore, none of the conventional high-temperature methods is applicable to grow the single crystals. To overcome this problem, we have developed a softchemical hydrothermal ion-exchange method capable of producing high-quality sizable single crystals of ( $\mathrm{Li}, \mathrm{Fe})$ OHFeSe [58]. Figure 1 schematically illustrates the hydrothermal ion-exchange process. For the hydrothermal ion-exchange reaction, large and high-quality $\mathrm{K}_{0.8} \mathrm{Fe}_{1.6} \mathrm{Se}_{2}$ crystal is used as a kind of matrix. The structure of $\mathrm{K}_{0.8} \mathrm{Fe}_{1.6} \mathrm{Se}_{2}$ is formed by an alternative stacking of $\mathrm{K}$-layer and FeSe-tetrahedron-block similar to the target compound. The $\mathrm{K}$ ions in $\mathrm{K}_{0.8} \mathrm{Fe}_{1.6} \mathrm{Se}_{2}$ are completely de-intercalated during the hydrothermal process. Simultaneously, the ( $\mathrm{Li}, \mathrm{Fe}$ ) $\mathrm{OH}$-blocks constructed by ions from the hydrothermal solution are intercalated into the matrix, and the ordered vacant $\mathrm{Fe}$-sites
(20\% in amount) originally in the matrix $\mathrm{Fe}_{0.8}$ Se-blocks are almost occupied. A series of large and high-quality ( $\mathrm{Li}, \mathrm{Fe}$ ) OHFeSe single crystals [60] are thus derived. The derived ( $\mathrm{Li}$, $\mathrm{Fe}) \mathrm{OHFeSe}$ single crystal almost inherits the original shape of the matrix (insets of Figures $\mathbf{1 B}, \mathbf{C}$ ). Inspired by the successful hydrothermal ion-exchange method for the single crystals, we have further invented a hydrothermal epitaxial film technique to fabricate a series of high-quality single-crystalline films of undoped [16, 66] and Mn-doped [17] (Li, Fe)OHFeSe, showing an optimal zero-resistivity $T_{c}=42.4 \mathrm{~K}$. The high-quality ( $\mathrm{Li}, \mathrm{Fe}$ ) OHFeSe films has enabled a systematic study of the superconducting and normal-state properties [66].

By modifying the hydrothermal reaction conditions, we have also developed a hydrothermal ion-deintercalation (HID) method, as illustrated in Figure 2. The atomic ratio of the FeSe-blocks can be continuously tuned by the HID process, yielding a series of non-stoichiometric $\mathrm{Fe}_{1-x} \mathrm{Se}$ single crystals at various charge-doping levels $[9,61,62]$. FeSe crystals used to be grown by chemical-vapor-transport [86, 87], flux-free floatingzone [88], and flux solution methods. These methods are hard to tune the chemical stoichiometry.

\section{ELECTRONIC AND SUPERCONDUCTING PROPERTIES STUDIED IN THE HYDROTHERMAL SINGLE CRYSTALS AND FILMS}

Now we briefly review our recent year's studies of the series of FeSe-based single crystals and films grown by the hydrothermal methods.

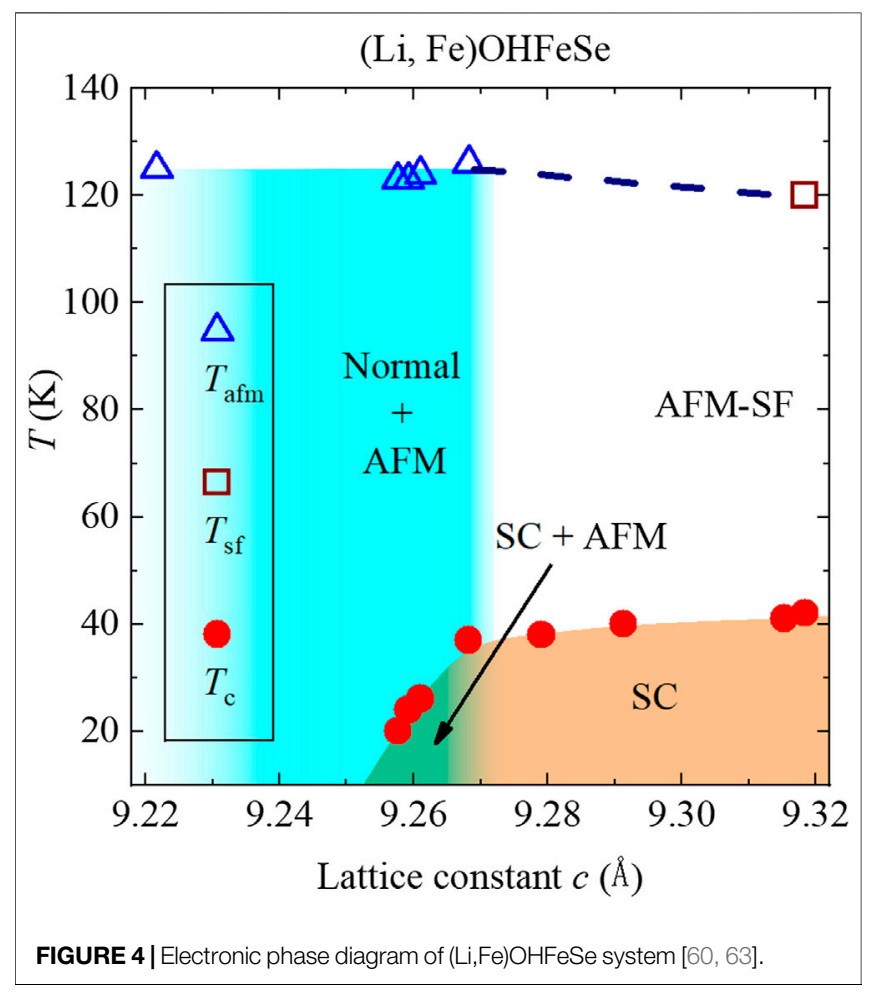


A

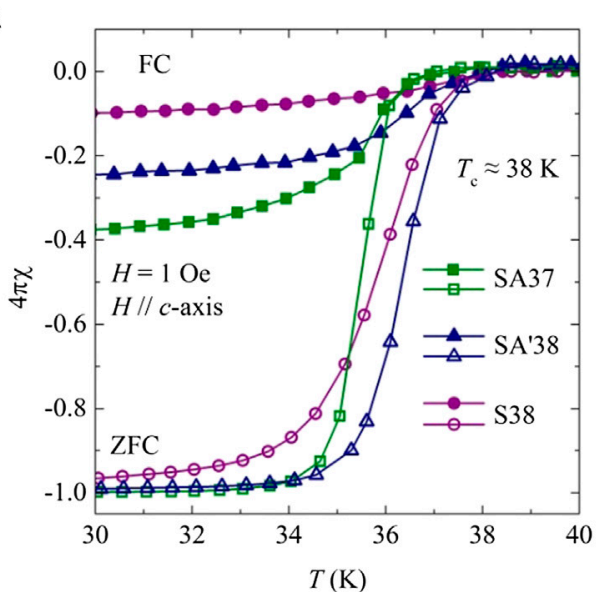

C

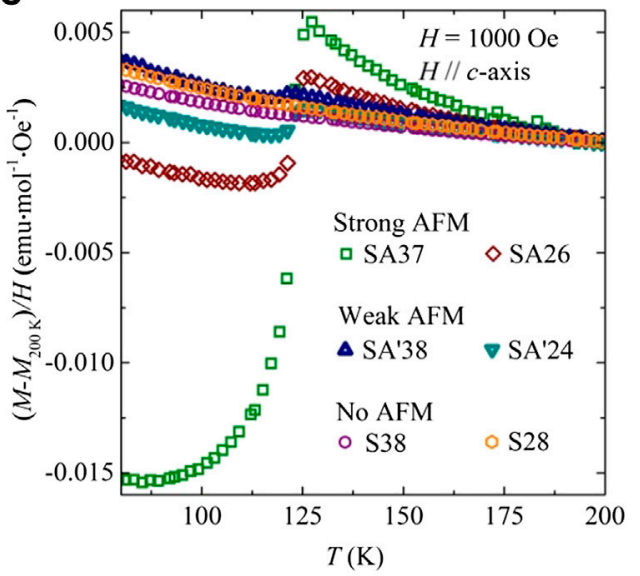

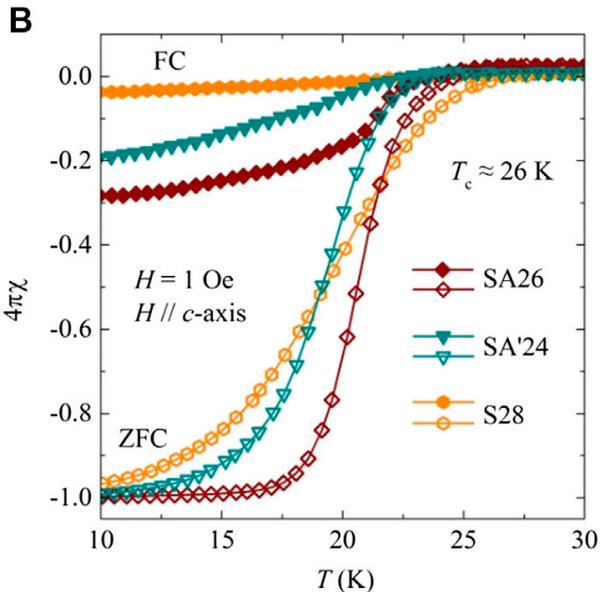

D

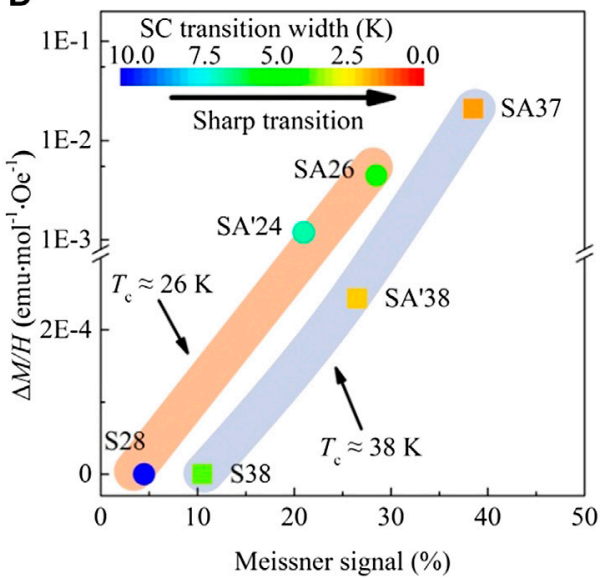

FIGURE $\mathbf{5}$ | (A,B) Temperature dependence of static magnetic susceptibility near the superconducting transitions, for the two sets of superconducting (Li,Fe) OHFeSe single crystals. (C) Antiferromagnetic (AFM) transition at $\sim 125 \mathrm{~K}$ is detectable for the superconducting (Tc < $38 \mathrm{~K}$ ) samples and non-superconducting samples. (D) The corresponding AFM signal size and the SC Meissner signal size are positively correlated (60).

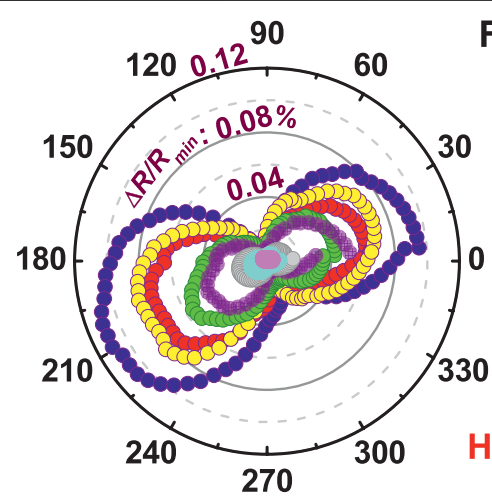

\section{FeSe (001)}

- $11 \mathrm{~K}$

- $15 \mathrm{~K}$

- $20 \mathrm{~K}$

- $25 \mathrm{~K}$

- $30 \mathrm{~K}$

- $40 \mathrm{~K}$

- $50 \mathrm{~K}$

$60 \mathrm{~K}$

$\mathrm{H}=9 \mathrm{~T}, \mathrm{H} / / \mathrm{ab}$

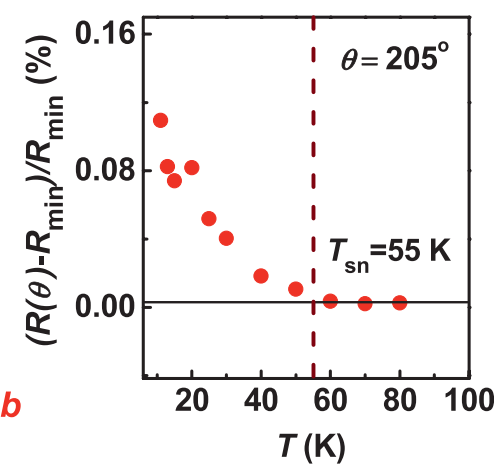

FIGURE 6 | Temperature dependences of the angular-dependent magnetoresistance of FeSe crystal $\left(T_{\mathrm{C}}=7.6 \mathrm{~K}\right)$, showing the twofold rotational symmetry below $T_{\text {sn }} \sim 55 \mathrm{~K}$ [64]. 

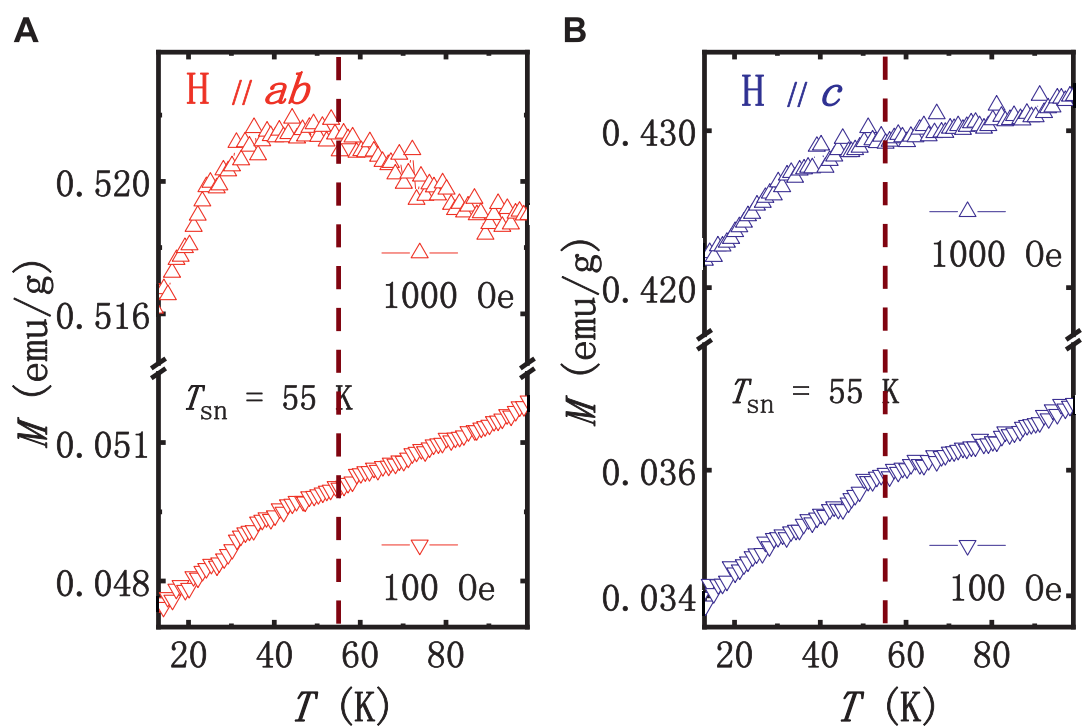

FIGURE 7 |Temperature dependence of the static magnetization around $T_{\mathrm{sn}}$ under the in-plane and out-of-plane fields for the FeSe crystal shown in Figure 6 [61].

\section{Strong Electronic Two-Dimensionality in High- $T_{c}(L i, F e) O H F e S e$ Single Crystal}

Figure $3 \mathbf{A}$ shows the temperature dependence of the in-plane resistivity, $\rho_{\mathrm{ab}}$, for the high- $T_{\mathrm{c}}(42 \mathrm{~K})\left(\mathrm{Li}_{0.84} \mathrm{Fe}_{0.16}\right) \mathrm{OHFe}_{0.98} \mathrm{Se}$ single crystal [58], which displays a metallic behavior over the whole measuring temperature range in the normal state. As a measure of the charge transport anisotropy, the ratio of the outof-plane to in-plane resistivity, $\rho_{\mathrm{c}} / \rho_{\mathrm{ab}}$, was found to increase with lowering temperature and reach a high value of 2,500 at $50 \mathrm{~K}$. It is obvious that the normal-state electronic property turns out to be

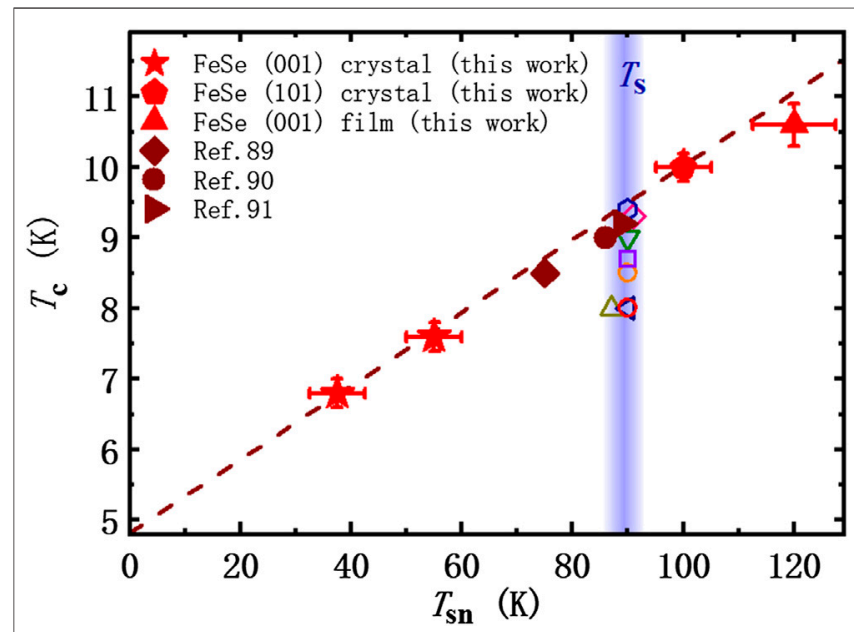

FIGURE 8 | The universal linear relationship between the superconducting transition temperature $\left(T_{\mathrm{C}}\right)$ and the field-induced spinnematic ordering temperature $\left(T_{\mathrm{sn}}\right)$ among various FeSe samples (the solid symbols) [64]. The hollow symbols in the vertical blue-shaded area represent the structure phase transition temperatures by the $x$-ray or neutron diffractions on various FeSe samples of different Tc's [30, 52, 55, 86, 92-94]. highly two dimensional just above $T_{\mathrm{c}}$. Shown in Figure $3 \mathrm{C}$ is the temperature dependence of static magnetic susceptibility, which is slightly dependent on the magnitude of the applied field. In the higher temperature range, all the data can be fitted to a modified Curie-Weiss law $\chi_{\mathrm{m}}=\chi_{0}+\chi_{\mathrm{CW}}$ (the solid lines), where $\chi_{0}$ is the Pauli paramagnetic contribution from itinerant charge carriers. A deviation from the Curie-Weiss law is clearly visible below a characteristic $T^{*}(\sim 120 \mathrm{~K})$ for a dip-like $T$-dependence of the Hall coefficient (Figure 3B), coinciding with the upturn in Hall coefficient and the change in resistivity behavior. From the Hall$\operatorname{dip} T^{\star}$ down to the superconducting $T_{\mathrm{c}}$, both the extracted ironplane magnetic susceptibility (with the Curie-Weiss term subtracted; inset of Figure 3C) and the in-plane resistivity (inset of Figure 3A) exhibit a linear temperature dependence, suggesting the presence of two-dimensional antiferromagnetic spin fluctuations in the iron planes.

\section{Phase Diagram and Electronic Phase Separation of (Li,Fe)OHFeSe System}

The first phase diagram of ( $\mathrm{Li}, \mathrm{Fe}) \mathrm{OHFeSe}$ system [63] was based on the powder samples. In a subsequent work [60], we established a more complete phase diagram for the system (Figure 4), based on a series of the hydrothermal single crystals in the superconducting (SC) and non-superconducting regimes. In some of the SC samples $\left(T_{\mathrm{c}}<\right.$ $\sim 38 \mathrm{~K}$, cell parameter $c<\sim 9.27 \AA$ ), we observed a strong drop in the magnetization at an almost constant temperature scale $T_{\text {afm }} \sim 125 \mathrm{~K}$ (Figure 5C), indicating the occurrence of antiferromagnetism well above $T_{c}$. Our analysis of electron energy-loss spectroscopy combined with selected-area electron diffraction confirmed the absence of magnetic impurity phases such as $\mathrm{Fe}_{3} \mathrm{O}_{4}$ [60]. Therefore, the antiferromagnetic signal is intrinsic to $(\mathrm{Li}, \mathrm{Fe}) \mathrm{OHFeSe}$ system. Moreover, a positive correlation between the sizes of the antiferromagnetic signal and the Meissner signal was observed 


\section{A}
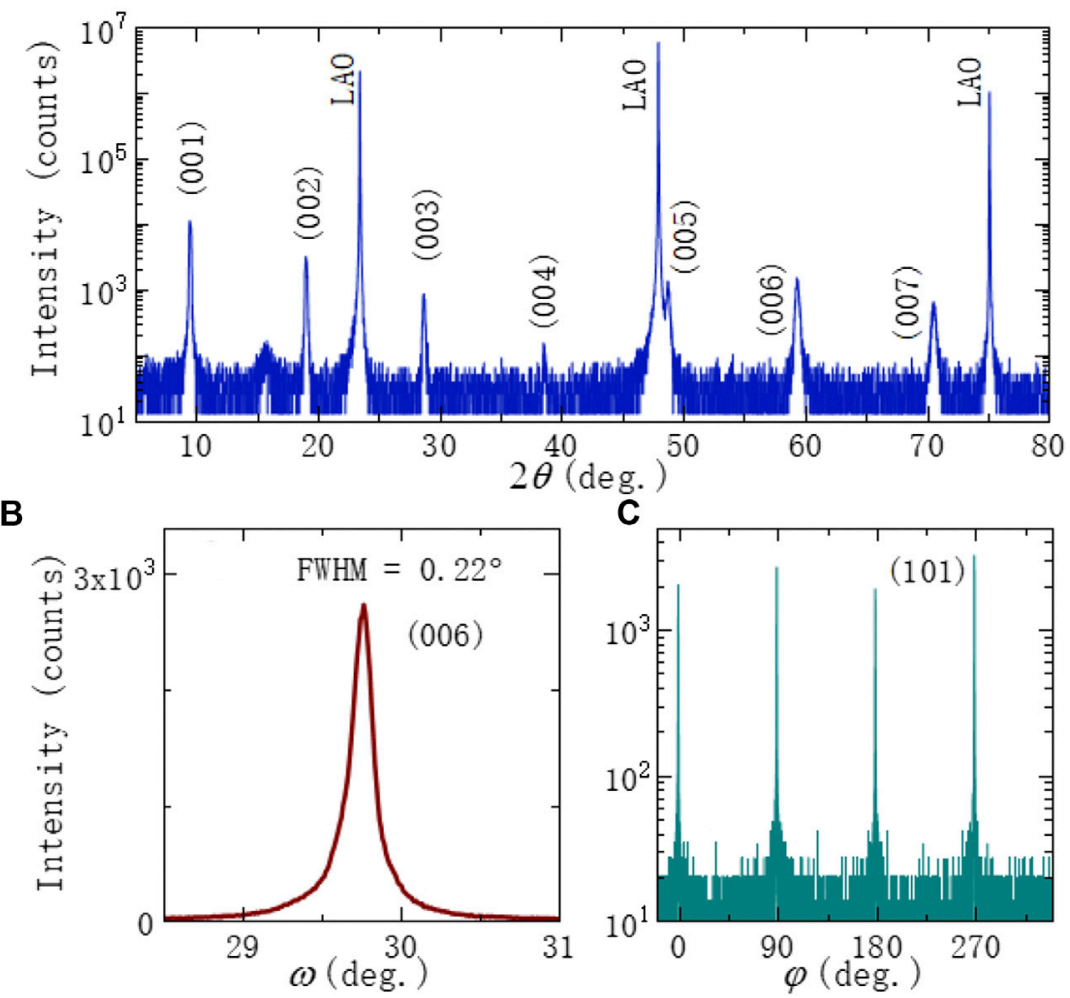

FIGURE 9|XRD characterizations of the ( $\mathrm{Li}, \mathrm{Fe}) \mathrm{OHFeSe}$ film on the $\mathrm{LaAlO}_{3}(\mathrm{LAO})$ substrate. (A) The two theta scan detects only $(00 /)$ peaks. (B) The rocking curve of (006) reflection with an FWHM of $0.22^{\circ}$. (C) The $\phi$-scan of the (101) plane. The 4-fold symmetry reveals an excellent epitaxial growth [16].

(Figures 5D). These experimental results demonstrate the coexistence of an antiferromagnetic state with the superconducting state in $(\mathrm{Li}, \mathrm{Fe}) \mathrm{OHFeSe}$ at $T_{\mathrm{c}}<\sim 38 \mathrm{~K}$ and $c<\sim 9.27 \AA$. Such coexistence can be explained by electronic phase separation [60], similar to the cases of high- $T_{\mathrm{c}}$ cuprates and iron arsenides. Therefore the electronic phase diagram shown in Figure 4 provides more information about the electronic states in ( $\mathrm{Li}, \mathrm{Fe}) \mathrm{OHFeSe}$ system.
The Link Between the Superconducting and Normal-State Properties in $\mathrm{Fe}_{1-x} \mathrm{Se}$ Single

\section{Crystals}

The in-plane angular-dependent magnetoresistance (AMR) in the normal state was measured for the hydrothermal $\mathrm{Fe}_{1-x} \mathrm{Se}$ single crystals [64]. Figure 6 shows the AMR at a $9 \mathrm{~T}$ field for a representative sample with $T_{\mathrm{c}}=7.6 \mathrm{~K}$. The AMR displays a two-
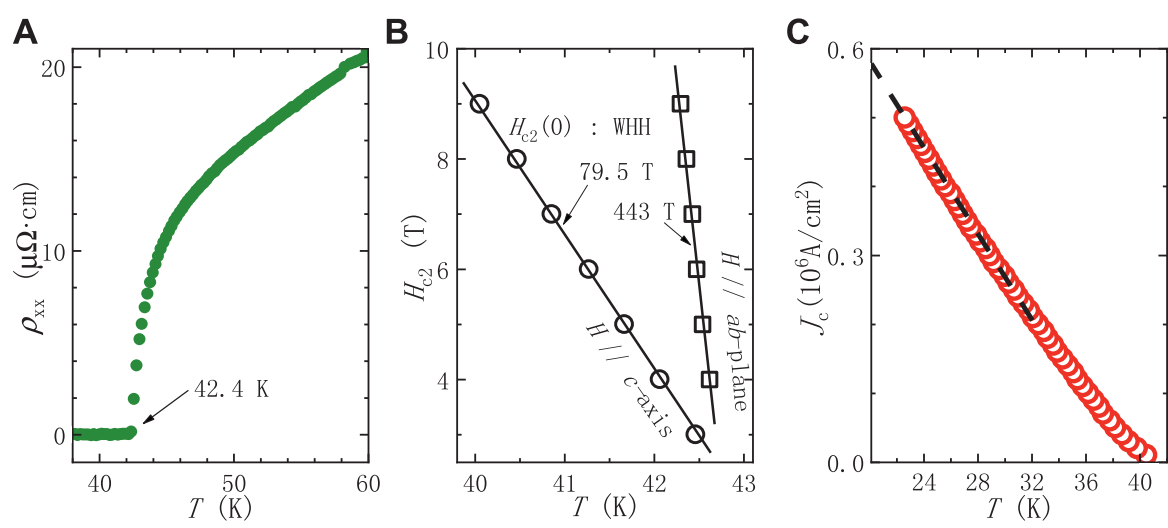

FIGURE 10| High superconducting critical parameters for (Li,Fe)OHFeSe film. (A) Temperature dependence of in-plane resistivity, with the onset of zero resistivity at $42.4 \mathrm{~K}$. (B) Temperature dependence of $\mathrm{H}_{\mathrm{c} 2}$ along the $\mathrm{c}$-axis (circle) and within the ab plane (square). (C) The temperature dependence of $J_{\mathrm{c}}$, exceeding $0.5 \mathrm{MA} / \mathrm{cm}^{2}$ at $20 \mathrm{~K} \mathrm{[16].}$ 


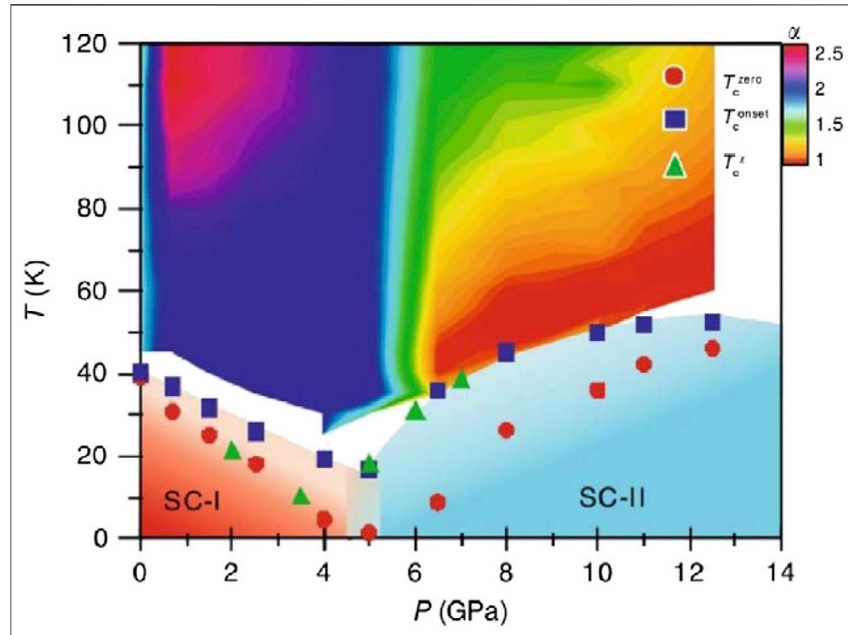

FIGURE 11 | Temperature-pressure phase diagram of $\left(\mathrm{Li}_{0.84} \mathrm{Fe}_{0.16}\right)$ $\mathrm{OHFe}_{0.98} \mathrm{Se}$ single crystal [70]. Pressure-dependence of $T_{\mathrm{C}}$ and contour plot of the normal-state resistivity exponent $\alpha$ are shown up to $12.5 \mathrm{GPa}$.

fold rotational symmetry emerging below a characteristic temperature $T_{\text {sn }} \sim 55 \mathrm{~K}$. This anisotropy in AMR is enhanced with decreasing temperature (left panel of Figure 6). This enhancement in charge scatterings was also observed in the temperature-dependent magnetoresistance by an earlier study [89]. Moreover, a downward curvature starting below $T_{\mathrm{sn}} \sim 55 \mathrm{~K}$ was observed in our sample in the static magnetization under an in-plane magnetic field of $0.1 \mathrm{~T}$ (Figure 7A) [61]. Such a feature is strongly dependent on the magnitude and direction of the applied field (Figures 7A vs 7B). This suggests that the strong quantum spin frustrations predominate in the iron planes. Although the orbitalnematic order associated with the structural transition at $T_{\mathrm{s}} \sim 90 \mathrm{~K}$ is also of a two-fold rotational symmetry, the obvious downward feature of in-plane static magnetization below $T_{\mathrm{sn}} \sim 55 \mathrm{~K}$, which is far below $T_{\mathrm{s}}$, suggests that the fourfold-rotational-symmetry breaking identified by our AMR measurements is closely related to the frustrated spins with anisotropic magnetic fluctuations. Therefore, a field-induced nematic state of a spin origin emerges below $T_{\mathrm{sn}}$.

By summarizing all the data of our samples, we found a remarkable linear relationship between $T_{\mathrm{c}}$ and $T_{\mathrm{sn}}$, as shown in Figure 8. Moreover, the related data of $T_{\mathrm{c}}$ and $T_{\mathrm{sn}}$ available from literature [89-91] also well satisfy this linear relationship. Namely, the linear relationship between superconducting $T_{c}$ and characteristic $T_{\text {sn }}$ of the field-induced spin-nematic state was observed to cover a wide range from far below to beyond $T_{\mathrm{s}}$. This further suggests that the superconductivity is more likely related to the anisotropic magnetic fluctuations. These results of prototypal FeSe system are consistent with those of intercalated high- $T_{\mathrm{c}}(\mathrm{Li}, \mathrm{Fe}) \mathrm{OHFeSe}$ presented above. It needs to be emphasized that, for nearly stoichiometric FeSe samples with a constant $T_{\mathrm{c}} \sim 9.5 \mathrm{~K}$, both the spin-nematic ordering and orbital-nematic ordering (associated with the structural transition) happen to coincide with each other at $\sim 90 \mathrm{~K}$, as shown in Figure 8. So it is difficult to distinguish these different ordering states in such samples. Our samples with different $T_{\mathrm{c}}$ 's enable the disentanglement of the different states.
Most recently, we have studied the doping dependences of electronic correlation effect [9] and upper critical field behavior [62] in a series of hydrothermal $\mathrm{Fe}_{1-x} \mathrm{Se}$ single crystals. Particularly in these binary $\mathrm{Fe}_{\mathbf{1}-\boldsymbol{x}} \mathrm{Se}$ samples, the charge-doping level can be tuned simply by the non-stoichiometric $x$, from a strong electron dominance at $x \sim 0$ to a strong hole dominance at higher $x$ values. Importantly, we find that superconductivity of FeSe system emerges from the strongly correlated, holedominated $\mathrm{Fe}_{1-x} \mathrm{Se}$ as the non-stoichiometry is reduced to $x \sim$ $5.3 \%$ [9]. Interestingly, such an $x$ threshold for superconductivity of the prototypal FeSe is similar to that $\left(x \sim 5 \%\right.$ [65]) for high- $T_{c}$ superconductivity of the intercalated ( $\mathrm{Li}, \mathrm{Fe}) \mathrm{OHFeSe}$ sharing the common superconducting FeSe-blocks.

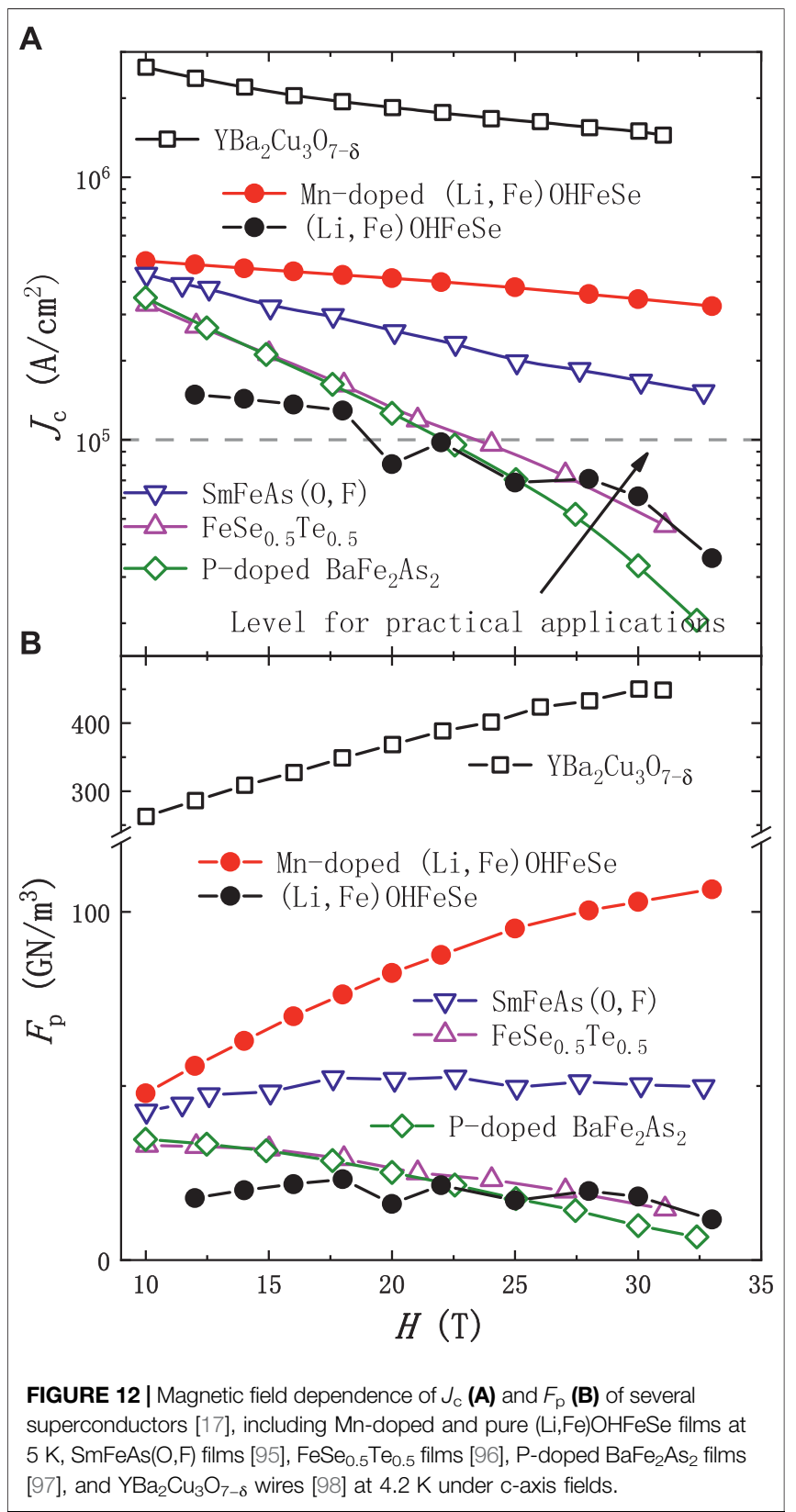




\section{High Superconducting Critical Parameters of Un-Doped and Mn-Doped (Li,Fe)OHFeSe Crystals and Films}

Figure 9 shows the $\mathrm{x}$-ray diffraction characterization of a representative ( $\mathrm{Li}, \mathrm{Fe}) \mathrm{OHFeSe}$ film sample hydrothermally grown on $\mathrm{LaAlO}_{3}$ substrate [16]. The observation of only (00l) reflections indicates a single preferred (001) orientation (Figure 9A). Shown in Figure 9B is the double-crystal $x$-ray rocking curve for the (006) Bragg reflection, with a small FWHM of $0.22^{\circ}$. To our knowledge, this is the best FWHM value observed so far among various iron-based superconductor crystals and films, indicating a high sample quality. The $\emptyset$-scan of (101) plane shown in Figure 9C exhibits four successive peaks with an equal interval of $90^{\circ}$, consistent with the $C_{4}$ symmetry of the $(\mathrm{Li}, \mathrm{Fe})$ $\mathrm{OHFeSe}$ film. These results clearly demonstrate an excellent inplane orientation and epitaxial growth.

High-quality superconducting films can play an important role in the application. Besides the high sample quality, the $(\mathrm{Li}, \mathrm{Fe})$ $\mathrm{OHFeSe}$ films also display excellent superconducting properties. The temperature dependence of in-plane resistivity is shown in Figure $10 \mathrm{~A}$, with a superconducting zero-resistivity temperature up to $42.4 \mathrm{~K}$. Figure 10B is the temperature dependences of upper critical field $H_{\mathrm{c} 2}$ derived from systematic measurements of the inplane and out-of-plane magnetoresistance. Based on $\mathrm{WHH}$ (Werthamer-Helfand-Hohenberg) model, the values of $H_{\mathrm{c} 2}(0)$ are estimated as 79.5 and $443 \mathrm{~T}$ at magnetic fields perpendicular and parallel to the $a b$ plane, respectively. Moreover, a large critical current density $J_{\mathrm{c}}>0.5 \mathrm{MA} / \mathrm{cm}^{2}$ was achieved at $\sim 20 \mathrm{~K}$, as shown in Figure 10C. The high superconducting critical parameters are important for practical application. Additionally, as seen from Figure 11, the critical temperature $T_{\mathrm{c}}$ of $\left(\mathrm{Li}_{0.84} \mathrm{Fe}_{0.16}\right) \mathrm{OHFe}_{0.98} \mathrm{Se}$ single crystal can be further raised up to a value $>50 \mathrm{~K}$ under a pressure of $12.5 \mathrm{GPa}$ in the superconducting phase II (SC-II) region. The SC-II phase develops with pressure at a critical $P_{\mathrm{c}}=$ $5 \mathrm{GPa}$, as the superconducting phase I (SC-I) is gradually suppressed.

Very recently, we have successfully doped $\mathrm{Mn}$ into ( $\mathrm{Li}, \mathrm{Fe})$ OHFeSe films [17]. As seen from Figure 12A, the $J_{c}$ value of high$T_{\mathrm{c}}$ (Li,Fe)OHFeSe film is strongly enhanced by one order of magnitude, from the undoped 0.03 to $\mathrm{Mn}$-doped $0.32 \mathrm{MA} / \mathrm{cm}^{2}$ under $33 \mathrm{~T}$ at $5 \mathrm{~K}$. The vortex pinning force density $F_{\mathrm{p}}$ monotonically increases with field up to $106 \mathrm{GN} / \mathrm{m}^{3}$, shown in Figure 12B. To the best of our knowledge, these values are the records so far among all the iron-based superconductors. Such a superconducting $(\mathrm{Li}, \mathrm{Fe}) \mathrm{OHFeSe}$ film is not only important for the fundamental research, but also promising for high-field application.

\section{CONCLUSION}

High-quality single crystals and single-crystalline films of ironbased superconductors play an important role in both the basic research and potential application. However, for the FeSebased superconductor systems reviewed here, by the conventional high-temperature growth it is either hard to obtain the single crystals and films, or not easy to tune the electronic properties. These problems can be overcome by our recently developed soft-chemical hydrothermal growth methods, which are capable of producing the single crystals and films, and tuning the chemical stoichiometry thus the electronic properties. In addition, these methods may be applicable in other layered materials, providing a new route for the exploration of functional materials.

The successful crystal and film growth has enabled systematic studies of the FeSe-based superconductor systems. We have observed a strong electronic two-dimensionality towards $T_{c}$, and a nearly linear extracted magnetic susceptibility as well as a linear in-plane resistivity both emerging below a Hall-dip temperature $T^{\star}(\sim 120 \mathrm{~K})$, in high- $T_{\mathrm{c}}$ intercalated $(\mathrm{Li}, \mathrm{Fe}) \mathrm{OHFeSe}$ system. We have also observed a linear relationship between $T_{\mathrm{c}}$ and characteristic temperature $T_{\mathrm{sn}}$ of a field-induced spin nematicity in prototypal FeSe system. These results suggest the presence of magnetic fluctuations in the iron planes and their relevance to superconductivity. Importantly, we have found that superconductivity of the prototypal FeSe emerges from the strongly correlated, hole-dominated $\mathrm{Fe}_{1-x} \mathrm{Se}$ at a nonstoichiometric $x$ similar to that for the high- $T_{c}$ superconductivity of the FeSe-based intercalate of ( $\mathrm{Li}, \mathrm{Fe}) \mathrm{OHFeSe}$. An electronic phase diagram has been established for ( $\mathrm{Li}, \mathrm{Fe}) \mathrm{OHFeSe}$ system, with the observed coexistence of antiferromagnetism and superconductivity explained by electronic phase separation. On the other hand, the high superconducting critical current density achieved in Mn-doped high- $T_{\mathrm{c}}(\mathrm{Li}, \mathrm{Fe}) \mathrm{OHFeSe}$ film is promising for high-field application. These FeSe-based superconductor systems deserve further experimental and theoretical studies, in both aspects of the underlying physics and potential application.

\section{AUTHOR CONTRIBUTIONS}

All authors listed have made a substantial, direct, and intellectual contribution to the work and approved if for publication.

\section{FUNDING}

This work was supported by National Natural Science Foundation of China (Nos. 11834016 and 11888101), the National Key Research and Development Program of China (Grant Nos. 2017YFA0303003, 2016YFA0300300), and the Strategic Priority Research Program and Key Research Program of Frontier Sciences of the Chinese Academy of Sciences (Grant Nos. XDB25000000, QYZDY-SSW-SLH001).

\section{ACKNOWLEDGMENTS}

We are very thankful to all our collaborators for their valuable scientific contributions in the past 6 years, especially Huaxue Zhou, Dongna Yuan, Yulong Huang, Yiyuan Mao, Shunli Ni, Jinpeng Tian, Dong Li, and Peipei Shen for sample preparation and characterization. We are also very grateful to Kui Jin, Jie 
Yuan, Wei $\mathrm{Hu}$, and Zhongpei Feng for electrical transport measurements and insightful discussions; Jinguang Cheng and Jianping Sun for high-pressure research; Zian Li, Huaixin Yang, and Jianqi Li for TEM and EELS studies; Guangming Zhang and Zhenyu Zhang for theoretical support; Xingjiang Zhou and Lin

\section{REFERENCES}

1. Kamihara $\mathrm{Y}$, Watanabe $\mathrm{T}$, Hirano $\mathrm{M}$, Hosono $\mathrm{H}$. Iron-based layered superconductor $\mathrm{La}[\mathrm{O} 1-\mathrm{xFx}] \mathrm{FeAs}(\mathrm{x}=0.05-0.12)$ with $\mathrm{Tc}=26 \mathrm{~K}$. J Am Chem Soc (2008) 130, 3296. doi:10.1021/ja800073m

2. Johnston DC. The puzzle of high temperature superconductivity in layered iron pnictides and chalcogenides. Adv Phys (2010) 59, 803. doi:10.1080/00018732. 2010.513480

3. Mazin II. Superconductivity gets an iron boost. Nature (2010) 464, 183. doi:10. 1038/nature08914

4. Paglione J, Greene RL. High-temperature superconductivity in iron-based materials. Nat Phys (2010) 6, 645. doi:10.1038/nphys1759

5. Stewart GR. Superconductivity in iron compounds. Rev Mod Phys (2011) 83, 1589. doi:10.1103/revmodphys.83.1589

6. Dagotto E. Colloquium: the unexpected properties of alkali metal iron selenide superconductors. Rev Mod Phys (2013) 85, 849. doi:10.1103/revmodphys.85.849

7. Chen X, Dai P, Feng D, Xiang T, Zhang F-C. Iron-based high transition temperature superconductors. Natl Sci Rev (2014) 1, 371. doi:10.1093/nsr/ nwu007

8. Shibauchi T, Hanaguri T, Matsuda Y. Exotic superconducting states in FeSebased materials. J Phys Soc Jpn (2020) 89, 102002.

9. Ni SL, Sun JP, Liu SB, Yuan J, Yu L, Ma MW, et al. Emergence of superconductivity in strongly correlated hole-dominated Fe1-xSe. arXiv preprint arXiv:1912.12614 (2019)

10. Putti M, Pallecchi I, Bellingeri E, Cimberle MR, Tropeano M, Ferdeghini C, et al. New Fe-based superconductors: properties relevant for applications. Supercond Sci Technol (2010) 23, 034003. doi:10.1088/0953-2048/23/3/034003

11. Li Q, Si W, Dimitrov IK. Films of iron chalcogenide superconductors. Rep Prog Phys (2011) 74, 124510. doi:10.1088/0034-4885/74/12/124510

12. Ma Y. Progress in wire fabrication of iron-based superconductors. Supercond Sci Technol (2012) 25, 113001. doi:10.1088/0953-2048/25/11/113001

13. Haindl S, Kidszun M, Oswald S, Hess C, Büchner B, Kölling S, et al. Thin film growth of Fe-based superconductors: from fundamental properties to functional devices. A comparative review. Rep Prog Phys (2014) 77, 046502. doi:10.1088/0034-4885/77/4/046502

14. Hosono H, Tanabe K, Takayama-Muromachi E, Kageyama H, Yamanaka S, Kumakura $\mathrm{H}$, et al. Exploration of new superconductors and functional materials, and fabrication of superconducting tapes and wires of iron pnictides. Sci Technol Adv Mater (2015) 16, 033503. doi:10.1088/14686996/16/3/033503

15. Hänisch J, Iida K, Huehne R, Tarantini C. Fe-based superconducting thin films-preparation and tuning of superconducting properties. Supercond Sci Technol (2019) 32, 093001. doi:10.1088/1361-6668/ablc00

16. Huang Y, Feng Z, Ni S, Li J, Hu W, Liu S, et al. Superconducting (Li,Fe)OHFeSe film of high quality and high critical parameters. Chin Phys Lett (2017) 34, 077404. doi:10.1088/0256-307x/34/7/077404

17. Li D, Yuan J, Shen P, Xi C, Tian J, Ni S, et al. Giant enhancement of critical current density at high field in superconducting $(\mathrm{Li}, \mathrm{Fe}) \mathrm{OHFeSe}$ films by $\mathrm{Mn}$ doping. Supercond Sci Technol (2019) 32, 12LT01. doi:10.1088/1361-6668/ ab4e7c

18. Wang D, Kong L, Fan P, Chen H, Zhu S, Liu W, et al. Evidence for Majorana bound states in an iron-based superconductor. Science (2018) 362, 333. doi:10. 1126/science.aaol797

19. Zhang P, Yaji K, Hashimoto T, Ota Y, Kondo T, Okazaki K, et al. Observation of topological superconductivity on the surface of an iron-based superconductor. Science (2018) 360, 182. doi:10.1126/science.aan4596

20. Liu Q, Chen C, Zhang T, Peng R, Yan Y-J, Wen C-H-P, et al. Robust and clean Majorana zero mode in the vortex core of high-temperature superconductor
Zhao for ARPES studies; Donglai Feng and Tong Zhang for STM studies; Li Pi, Chuanying Xi, Zhaosheng Wang, and J. Wosnitza for high-field studies; Rustem Khasanov, Zurab Guguchia, and Alex Amato for $\mu$ SR studies. We also thank Ping Zheng, Shaokui $\mathrm{Su}$, and Lihong Yang for technical supports.

(Li0.84Fe0.16)OHFeSe. Phys Rev X (2018) 8, 041056. doi:10.1103/physrevx.8. 041056

21. Chen C, Liu Q, Zhang TZ, Li D, Shen PP, Dong XL, et al. Quantized conductance of Majorana zero mode in the vortex of the topological superconductor (Li0.84Fe0.16)OHFeSe. Chin Phys Lett (2019) 36, 057403. doi:10.1088/0256-307x/36/5/057403

22. Cao C, Hirschfeld PJ, Cheng H-P. Proximity of antiferromagnetism and superconductivity inLaFeAsO1-xFx: effective Hamiltonian fromab initiostudies. Phys Rev B (2008) 77, 220506R. doi:10.1103/physrevb.77.220506

23. Si Q, Abrahams E. Strong correlations and magnetic frustration in the high Tc iron pnictides. Phys Rev Lett (2008) 101, 076401. doi:10.1103/physrevlett.101. 076401

24. Lu Z-Y, Ma F, Xiang T. Pnicogen-bridged antiferromagnetic superexchange interactions in iron pnictides. J Phys Chem Solid (2011) 72, 319. doi:10.1016/j. jpcs.2010.10.065

25. Fernandes RM, Chubukov AV, Schmalian J. What drives nematic order in iron-based superconductors? Nat Phys (2014) 10, 97. doi:10.1038/nphys2877

26. Hsu F-C, Luo J-Y, Yeh K-W, Chen T-K, Huang T-W, Wu PM, et al. Superconductivity in the PbO-type structure-FeSe. Proc Natl Acad Sci U S A (2008) 105, 14262. doi:10.1073/pnas.0807325105

27. Mizuguchi Y, Tomioka F, Tsuda S, Yamaguchi T, Takano Y. Superconductivity at $27 \mathrm{~K}$ in tetragonal FeSe under high pressure. Appl Phys Lett (2008) 93, 152505. doi:10.1063/1.3000616

28. Margadonna S, Takabayashi Y, Ohishi Y, Mizuguchi Y, Takano Y, Kagayama T, et al. Pressure evolution of the low-temperature crystal structure and bonding of the superconductor FeSe $\left(\mathrm{T}_{c}=37 \mathrm{~K}\right)$. Phys Rev B (2009) 80, 064506. doi:10.1103/physrevb.80.064506

29. Medvedev S, McQueen TM, Troyan IA, Palasyuk T, Eremets MI, Cava RJ, et al. Electronic and magnetic phase diagram of $\beta$-Fe1.01Se with superconductivity at $36.7 \mathrm{~K}$ under pressure. Nat Mater (2009) 8, 630. doi:10.1038/nmat2491

30. Kothapalli K, Bohmer AE, Jayasekara WT, Ueland BG, Das P, Sapkota A, et al. Strong cooperative coupling of pressure-induced magnetic order and nematicity in FeSe. Nat Commun (2016) 7, 12728. doi:10.1038/ncomms12728

31. Sun JP, Matsuura K, Ye GZ, Mizukami Y, Shimozawa M, Matsubayashi K, et al. Dome-shaped magnetic order competing with high-temperature superconductivity at high pressures in FeSe. Nat Commun (2016) 7, 12146. doi:10.1038/ncomms12146

32. Khasanov R, Guguchia Z, Amato A, Morenzoni E, Dong X, Zhou F, et al. Pressure-induced magnetic order in FeSe: a muon spin rotation study. Phys Rev B (2017) 95, 180504R. doi:10.1103/physrevb.95.180504

33. Khasanov R, Fernandes RM, Simutis G, Guguchia Z, Amato A, Luetkens H, et al. Magnetic tricritical point and nematicity in FeSe under pressure. Phys Rev B (2018) 97, 224510. doi:10.1103/physrevb.97.224510

34. Lei B, Cui JH, Xiang ZJ, Shang C, Wang NZ, Ye GJ, et al. Evolution of hightemperature superconductivity from a low-Tc phase tuned by carrier concentration in FeSe thin flakes. Phys Rev Lett (2016) 116, 077002. doi:10. 1103/physrevlett.116.077002

35. Shikama N, Sakishita Y, Nabeshima F, Katayama Y, Ueno K, Maeda A. Enhancement of superconducting transition temperature in electrochemically etched FeSe/LaAlO3 films. 13, 06982 (2020)

36. Guo J, Jin S, Wang G, Wang S, Zhu K, Zhou T, et al. Superconductivity in the iron selenide KxFe2Se2 (0 $\leq x \leq 1.0)$. Phys Rev B (2010) 82, 180520R. doi:10. 1103 /physrevb.82.180520

37. Fang M-H, Wang H-D, Dong C-H, Li Z-J, Feng C-M, Chen J, et al. Fe-based superconductivity with $\mathrm{T} \mathrm{c}=31 \mathrm{~K}$ bordering an antiferromagnetic insulator in (Tl,K) Fe x Se 2. Epl (2011) 94, 27009. doi:10.1209/0295-5075/94/27009

38. Wang AF, Ying JJ, Yan YJ, Liu RH, Luo XG, Li ZY, et al. Superconductivity at 32 K in single-crystalline RbxFe2-ySe2. Phys Rev B (2011) 83, 060512R. doi:10. 1103/physrevb.83.060512 
39. Ying TP, Chen XL, Wang G, Jin SF, Zhou TT, Lai XF, et al. Observation of superconductivity at 30 similar to $46 \mathrm{~K}$ in $\mathrm{AxFe} 2 \mathrm{Se} 2(\mathrm{~A}=\mathrm{Li}, \mathrm{Na}, \mathrm{Ba}, \mathrm{Sr}, \mathrm{Ca}, \mathrm{Yb}$, and Eu). Sci Rep (2012) 2, 426. doi:10.1038/srep00426

40. Zhang AM, Xia TL, Liu K, Tong W, Yang ZR, Zhang QM. Superconductivity at $44 \mathrm{~K}$ in K intercalated FeSe system with excess Fe. Sci Rep (2013) 3, 1216. doi:10.1038/srep01216

41. Lu XF, Wang NZ, Wu H, Wu YP, Zhao D, Zeng XZ, et al. Coexistence of superconductivity and antiferromagnetism in (Li0.8Fe0.2)OHFeSe. Nat Mater (2014) 14, 325. doi:10.1038/nmat4155

42. Krzton-Maziopa A, Pomjakushina EV, Pomjakushin VY, von Rohr F, Schilling A, Conder K. Synthesis of a new alkali metal-organic solvent intercalated iron selenide superconductor withTc $\approx 45 \mathrm{~K}$. J Phys-condens Mat (2012) 24, 382202. doi:10.1088/0953-8984/24/38/382202

43. Burrard-Lucas M, Free DG, Sedlmaier SJ, Wright JD, Cassidy SJ, Hara Y, et al. Enhancement of the superconducting transition temperature of FeSe by intercalation of a molecular spacer layer. Nat Mater (2013) 12, 15. doi:10. 1038/nmat3464

44. Sedlmaier SJ, CassidyMorris RG, Drakopoulos M, Reinhard C, Moorhouse SJ, et al. Ammonia-rich high-temperature superconducting intercalates of iron selenide revealed through time-resolved in Situ X-ray and neutron diffraction. J Am Chem Soc (2014) 136, 630. doi:10.1021/ja411624q

45. Scheidt EW, Hathwar VR, Schmitz D, Dunbar A, Scherer W, Mayr F, et al. Superconductivity at $\mathrm{Tc}=44 \mathrm{~K}$ in LixFe2Se2(NH3)y. Eur Phys J B (2012) 85, 279. doi:10.1140/epjb/e2012-30422-6

46. Ying T, Chen X, Wang G, Jin S, Lai X, Zhou T, et al. Superconducting phases in potassium-intercalated iron selenides. J Am Chem Soc (2013) 135, 2951. doi:10. $1021 /$ ja312705x

47. Sun S, Wang S, Rong Y, Lei H. Extreme anisotropy and anomalous transport properties of heavily electron doped $\mathrm{Li}-\mathrm{x}(\mathrm{NH} 3)(\mathrm{y}) \mathrm{Fe} 2 \mathrm{Se} 2$ single crystals. Phys Rev B (2017) 96, 064512. doi:10.1103/physrevb.96.064512

48. Noji T, Hatakeda T, Hosono S, Kawamata T, Kato M, Koike Y. Synthesis and post-annealing effects of alkaline-metal-ethylenediamine-intercalated superconductors $\mathrm{A}(\mathrm{C} 2 \mathrm{H} 8 \mathrm{~N} 2) \mathrm{Fe} 2-\mathrm{Se} 2(\mathrm{~A}=\mathrm{Li}, \mathrm{Na})$ with $\mathrm{Tc}=45 \mathrm{~K}$. PHYSICA C (2014) 504, 8. doi:10.1016/j.physc.2014.01.007

49. Wang Q-Y, Li Z, Zhang W-H, Zhang Z-C, Zhang J-S, Li W, et al. Interfaceinduced high-temperature superconductivity in single unit-cell FeSe films on $\mathrm{SrTiO}_{3}$. Chin Phys Lett (2012) 29, 037402. doi:10.1088/0256-307x/29/3/037402

50. He S, He J, Zhang W, Zhao L, Liu D, Liu X, et al. Phase diagram and electronic indication of high-temperature superconductivity at $65 \mathrm{~K}$ in single-layer FeSe films. Nat Mater (2013) 12, 605. doi:10.1038/nmat3648

51. Margadonna S, Takabayashi Y, McDonald MT, Kasperkiewicz K, Mizuguchi $\mathrm{Y}$, Takano Y, et al. Crystal structure of the new FeSe1-x superconductor. Chem Commun (Camb) (2008) 2008, 5607-5609. doi:10.1039/b813076k

52. McQueen TM, Williams AJ, Stephens PW, Tao J, Zhu Y, Ksenofontov V, et al. Tetragonal-to-orthorhombic structural phase transition at $90 \mathrm{~K}$ in the superconductor Fe1.01Se. Phys Rev Lett (2009) 103, 057002. doi:10.1103/ physrevlett.103.057002

53. Nakayama K, Miyata Y, Phan GN, Sato T, Tanabe Y, Urata T, et al. Reconstruction of band structure induced by electronic nematicity in an FeSe superconductor. Phys Rev Lett (2014) 113, 237001. doi:10.1103/ physrevlett.113.237001

54. Shimojima T, Suzuki Y, Sonobe T, Nakamura A, Sakano M, Omachi J, et al. Lifting of $\mathrm{xz} / \mathrm{yz}$ orbital degeneracy at the structural transition in detwinned FeSe. Phys Rev B (2014) 90, 121111R. doi:10.1103/physrevb.90.121111

55. Baek S-H, Efremov DV, Ok JM, Kim JS, van den Brink J, Büchner B. Orbitaldriven nematicity in FeSe. Nat Mater (2015) 14, 210. doi:10.1038/nmat4138

56. Niu XH, Peng R, Xu HC, Yan YJ, Jiang J, Xu DF, et al. Surface electronic structure and isotropic superconducting gap in $\left(\mathrm{Li}_{0.8} \mathrm{Fe}_{0.2}\right) \mathrm{OHFeSe}$. Phys Rev $B$ (2015) 92, 060504R. doi:10.1103/physrevb.92.060504

57. Zhao L, Liang A, Yuan D, Hu Y, Liu D, Huang J, et al. Common electronic origin of superconductivity in (Li, Fe)OHFeSe bulk superconductor and singlelayer FeSe/SrTiO3 films. Nat Commun (2016) 7, 10608. doi:10.1038/ncomms10608

58. Dong X, Jin K, Yuan D, Zhou H, Yuan J, Huang Y, et al. Li0.84Fe0.16) OHFe0.98Se superconductor: ion-exchange synthesis of large single-crystal and highly two-dimensional electron properties. Phys Rev B (2015) 92, 064515. doi:10.1103/physrevb.92.064515

59. Zhou H, Ni S, Yuan J, Li J, Feng Z, Jiang X, et al. Doping Mn into (Li1-xFex) OHFe1-ySe superconducting crystals via ion-exchange and ion-release/ introduction syntheses. Chin Phys B (2017) 26, 057402. doi:10.1088/16741056/26/5/057402

60. Mao YY, Li J, Huan YL, Yuan J, Li ZA, Chai K, et al. Electronic phase separation in iron selenide (Li,Fe)OHFeSe superconductor system. Chin Phys Lett (2018) 35, 057402. doi:10.1088/0256-307x/35/5/057402

61. Yuan D, Huang Y, Ni S, Zhou H, Mao Y, Hu W, et al. Synthesis of large FeSe superconductor crystals via ion release/introduction and property characterization. Chin Phys B (2016) 25, 077404. doi:10.1088/1674-1056/ 25/7/077404

62. Ni S, Hu W, Shen P, Wei Z, Liu S, Li D, et al. Different behavior of upper critical field in Fe 1- x Se single crystals. Chinese Phys. B (2019) 28, 127401. doi:10.1088/1674-1056/ab50b4

63. Dong X, Zhou H, Yang H, Yuan J, Jin K, Zhou F, et al. Phase diagram of (Li1xFex)OHFeSe: a bridge between iron selenide and arsenide superconductors. J Am Chem Soc (2015) 137, 66. doi:10.1021/ja511292f

64. Yuan D, Yuan J, Huang Y, Ni S, Feng Z, Zhou H, et al. Observation of Ising spin-nematic order and its close relationship to the superconductivity in $\mathrm{FeSe}$ single crystals. Phys Rev B (2016) 94, 060506R. doi:10.1103/physrevb.94.060506

65. Sun H, Woodruff DN, Cassidy SJ, Allcroft GM, Sedlmaier SJ, Thompson AL, et al. Soft chemical control of superconductivity in lithium iron selenide Hydroxides Li1-xFex(OH)Fe1-ySe. Inorg Chem (2015) 54, 1958. doi:10.1021/ ic5028702

66. Huang YL, Feng ZP, Yuan J, Hu W, Li J, Ni SL, et al. Matrix-assisted fabrication and exotic charge mobility of $(\mathrm{Li}, \mathrm{Fe}) \mathrm{OHFeSe}$ superconductor films. arXiv preprint arXiv:1711.02920 (2017)

67. Khasanov R, Zhou H, Amato A, Guguchia Z, Morenzoni E, Dong X, et al. Proximity-induced superconductivity within the insulating (Li0.84Fe0.16) OH layers in (Li0.84Fe0.16)OHFe0.98Se. Phys Rev B (2016) 93, 224512. doi:10. 1103/physrevb.93.224512

68. Smidman M, Pang GM, Zhou HX, Wang NZ, Xie W, Weng ZF, et al. Probing the superconducting gap structure of (Li1-xFex)OHFeSe. Phys Rev B (2017) 96, 014504. doi:10.1103/physrevb.96.014504

69. Wang Z, Yuan J, Wosnitza J, Zhou H, Huang Y, Jin K, et al. The upper critical field and its anisotropy in (Li1-xFex)OHFe1-ySe. J. Phys-condens. Mat (2017) 29, 025701. doi:10.1088/0953-8984/29/2/025701

70. Sun JP, Shahi P, Zhou HX, Huang YL, Chen KY, Wang BS, et al. Reemergence of high-T-c superconductivity in the (Li1-xFex)OHFe1-ySe under high pressure. Nat Commun (2018) 9, 380. doi:10.1038/s41467-018-02843-7

71. Xiao H, Hu T, Zhou HX, Li XJ, Ni SL, Zhou F, et al. Probing the anisotropy of (Li0.84Fe0.16)OHFe0.98Se by angular-dependent torque measurements. Phys Rev B (2020) 101, 184520. doi:10.1103/physrevb.101.184520

72. Du Z, Yang X, Lin H, Fang D, Guan D, Xing J, et al. Scrutinizing the double superconducting gaps and strong coupling pairing in $\left(\mathrm{Li}_{1-\mathrm{x}} \mathrm{Fe}_{\mathrm{x}}\right) \mathrm{OHFeSe}$. Nat Commun (2016) 7, 10565. doi:10.1038/ncomms10565

73. Zhou X, Borg CKH, Lynn JW, Saha SR, Paglione J, Rodriguez EE, et al. The preparation and phase diagrams of (7Li1-xFexOD)FeSe and ( $\mathrm{Li} 1-\mathrm{xFexOH})$ FeSe superconductors. J Mater Chem C (2016) 4, 3934. doi:10.1039/ c5tc04041h

74. Pan B, Shen Y, Hu D, Yu F, Park JT, Christianson AD, et al. Structure of spin excitations in heavily electron-doped Li0.8Fe $0.2 \mathrm{ODFeSe}$ superconductors. Nat Commun (2017) 8, 123. doi:10.1038/s41467-017-00162-x

75. Ma M, Wang L, Bourges P, Sidis YDanilkin SYuan L. Low-energy spin excitations in $\left(\mathrm{Li}_{0.8} \mathrm{Fe}_{0.2}\right)$ ODFeSe superconductor studied with inelastic neutron scattering. Phys Rev B (2017) 95, 100504R. doi:10.1103/physrevb. 95.100504

76. Ren M, Yan Y, Niu X, Tao R, Hu D, Peng R, et al. Superconductivity across Lifshitz transition and anomalous insulating state in surface $\mathrm{K}$-dosed $\left(\mathrm{Li}_{0.8} \mathrm{Fe}_{0.2} \mathrm{OH}\right) \mathrm{FeSe}$. Science Advances (2017) 3, el603238. doi:10.1126/ sciadv. 1603238

77. Sun Y, Pyon S, Yang R, Qiu X, Feng J, Shi Z, et al. Deviation from canonical collective creep behavior in $\mathrm{Li}_{0.8} \mathrm{Fe}_{0.2} \mathrm{OHFeSe}$. J Phys Soc Jpn (2019) 88, 034703. doi:10.7566/jpsj.88.034703

78. Yi X, Qin L, Xing X, Lin B, Li M, Meng Y, et al. Synthesis of (Li1-Fe)OHFeSe and FeSe single crystals without using selenourea via a hydrothermal method. J Phys Chem Solid (2020) 137, 109207. doi:10.1016/j.jpcs.2019.109207

79. Borg CKH, Zhou X, Eckberg C, Campbell DJ, Saha SR, Paglione J, et al. Strong anisotropy in nearly ideal tetrahedral superconducting FeS single crystals. Phys Rev B (2016) 93, 094522. doi:10.1103/physrevb.93.094522 
80. Yu G, Zhang GY, Ryu GH, Lin CT. Structure and superconductivity of (Li1-x $\mathrm{Fe} x$ )OHFeSe single crystals grown using A x Fe2-y Se2 (A = K, Rb, and Cs) as precursors. J Phys Condens Matter (2016) 28, 015701. doi:10.1088/0953-8984/ 28/1/015701

81. Lin H, Li Y, Deng Q, Xing J, Liu J, Zhu X, et al. Multiband superconductivity and large anisotropy in FeS crystals. Phys Rev B (2016) 93, 144505. doi:10. 1103/physrevb.93.144505

82. Yi X, Wang C, Tang Q, Peng T, Qiu Y, Xu J, et al. Vortex phase transition and anisotropy behavior of optimized (Li1-xFexOH)FeSe single crystals. Supercond Sci Technol (2016) 29, 105015. doi:10.1088/0953-2048/29/10/105015

83. Wilfong B, Zhou X, Zheng H, Babra N, Brown CM, Lynn JW, et al. Long-range magnetic order in hydroxide-layer-doped (Li1-x-yFexMnyOD)FeSe. Phys Rev Materials (2020) 4, 034803. doi:10.1103/physrevmaterials.4.034803

84. Lynn JW, Zhou X, Christopher K, Borg H, Saha SR, Paglione J, et al. Neutron investigation of the magnetic scattering in an iron-based ferromagnetic superconductor. Phys Rev B (2015) 92, 060510R. doi:10.1103/physrevb.92. 060510

85. Pachmayr U, Nitsche F, Luetkens H, Kamusella S, Brückner F, Sarkar R, et al. Coexistence of 3d-Ferromagnetism and Superconductivity in [(Li1-xFex) $\mathrm{OH}](\mathrm{Fel}-\mathrm{yLiy}) \mathrm{Se}$. Angew. Chem. Int. Edit (2015) 54, 293. doi:10.1002/anie. 201407756

86. Böhmer AE, Hardy F, Eilers F, Ernst D, Adelmann P, Schweiss P, et al. Lack of coupling between superconductivity and orthorhombic distortion in stoichiometric single-crystalline FeSe. Phys Rev B (2013) 87, 180505R. doi:10.1103/physrevb.87.180505

87. Chareev D, Osadchii E, Kuzmicheva T, Lin J-Y, Kuzmichev S, Volkova O, et al. Single crystal growth and characterization of tetragonal FeSe1-x superconductors. CrystEngComm (2013) 15, 1989. doi:10.1039/c2ce26857d

88. Ma M, Yuan D, Wu Y, Zhou H, Dong X, Zhou F. Flux-free growth of large superconducting crystal of FeSe by traveling-solvent floating-zone technique. Supercond Sci Technol (2014) 27, 122001. doi:10.1088/0953-2048/27/12/122001

89. Rößler S, Koz C, Lin J, Rößler UK, Frank S, Schwarz U, et al. Emergence of an incipient ordering mode in FeSe. Phys Rev B (2015) 92, 060505R. doi:10.1103/ physrevb.92.060505

90. Sun Y, Pyon S, Tamegai T. Electron carriers with possible Dirac-cone-like dispersion in FeSe1-xSx ( $\mathrm{x}=0$ and 0.14$)$ single crystals triggered by structural transition. Phys Rev B (2016) 93, 104502. doi:10.1103/physrevb.93.104502
91. Urata T, Tanabe Y, Huynh KK, Yamakawa Y, Kontani H, Tanigaki K. Superconductivity pairing mechanism from cobalt impurity doping in FeSe spin (s \pm ) or orbital (s++) fluctuation. Phys Rev B 93, 014507 (2016) doi:10. 1103/physrevb.93.014507

92. Rahn MC, Ewings RA, Sedlmaier SJ, Clarke SJ, Boothroyd AT. Strong $(\pi, 0)$ spin fluctuations in $\beta$-FeSe observed by neutron spectroscopy. Phys Rev $B$ (2015) 91, 180501R. doi:10.1103/physrevb.91.180501

93. Pachmayr U, Fehn N, Johrendt D. Structural transition and superconductivity in hydrothermally synthesized FeX $(X=S, S e)$. Chem. Commun (2016) 52, 194. doi:10.1039/c5cc07739g

94. Wang Q, Shen Y, Pan B, Hao Y, Ma M, Zhou F, et al. Strong interplay between stripe spin fluctuations, nematicity and superconductivity in FeSe. Nat Mater (2016) 15, 159. doi:10.1038/nmat4492

95. Bourges K, Haenisch J, Tarantini C, Kurth F, Jaroszynski J, Ueda S, et al. Oxypnictide $\mathrm{SmFeAs}(\mathrm{O}, \mathrm{F})$ superconductor: a candidate for high-field magnet applications. Sci Rep (2013) 3, 2139. doi:10.1038/srep02139

96. Si W, Han SJ, Shi X, Ehrlich SN, Jaroszynski J, Goyal A, et al. High current superconductivity in FeSe0.5Te0.5-coated conductors at 30 tesla. Nat Commun (2013) 4, 1347. doi:10.1038/ncomms 2337

97. Kurth F, Tarantini C, Grinenko V, Hänisch J, Jaroszynski J, Reich E, et al. Unusually high critical current of clean P-doped BaFe2As2 single crystalline thin film. Appl Phys Lett (2015) 106, 072602. doi:10.1063/1.4908257

98. Xu A, Jaroszynski JJ, Kametani F, Chen Z, Larbalestier DC, Viouchkov YL, et al. Angular dependence of Jc for YBCO coated conductors at low temperature and very high magnetic fields. Supercond Sci Technol (2010) 23, 014003. doi:10.1088/0953-2048/23/1/014003

Conflict of Interest: The authors declare that the research was conducted in the absence of any commercial or financial relationships that could be construed as a potential conflict of interest.

Copyright (C) 2020 Dong, Zhou and Zhao. This is an open-access article distributed under the terms of the Creative Commons Attribution License (CC BY). The use, distribution or reproduction in other forums is permitted, provided the original author(s) and the copyright owner(s) are credited and that the original publication in this journal is cited, in accordance with accepted academic practice. No use, distribution or reproduction is permitted which does not comply with these terms. 